\title{
The CRAN bayesvl package on T.C. İstanbul Gedik Üniversitesi
}

The Comprehensive R Archive Network (CRAN)

August 25, 2019

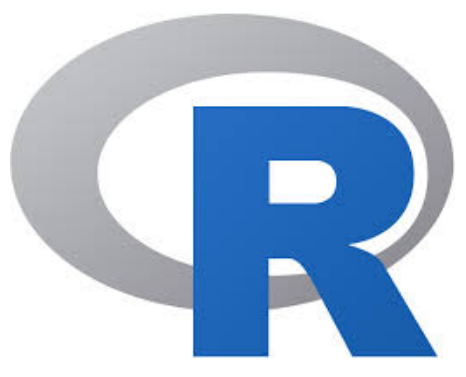

The R Core Team

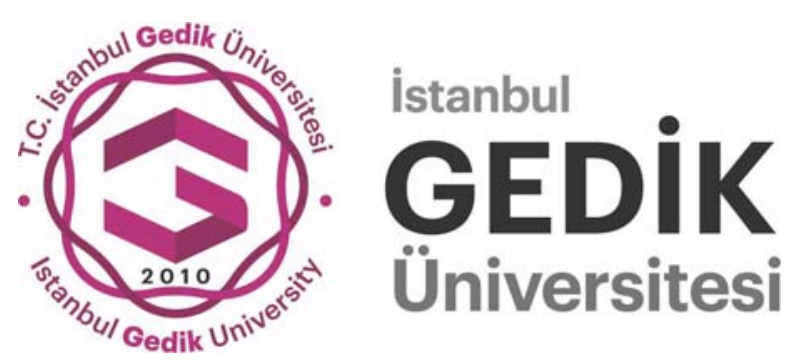

T.C. İstanbul Gedik Üniversitesi

https://cran.gedik.edu.tr/web/packages/bayesvl/index.html 


\section{bayesvl: Visually Learning the Graphical Structure of Bayesian Networks and Performing MCMC with 'Stan'}

Provides users with its associated functions for pedagogical purposes in visually learning Bayesian networks and Markov chain Monte Carlo (MCMC) computations. It enables users to:

a) Create and examine the (starting) graphical structure of Bayesian networks; b) Create random Bayesian networks using a dataset with customized constraints; c) Generate 'Stan' code for structures of Bayesian networks for sampling the data and learning parameters; d) Plot the network graphs; e) Perform Markov chain Monte Carlo computations and produce graphs for posteriors checks. The package refers to one reference item, which describes the methods and algorithms: Vuong, Quan-Hoang and La, Viet-Phuong (2019) < doi:10.31219/osf.io/w5dx6> The 'bayesvl' R package. Open Science Framework (May 18).

\section{Version: $\quad 0.8 .5$}

Depends: $\quad \mathrm{R}(\geq 3.4 .0)$, $\underline{\text { rstan }}(\geq 2.10 .0)$, $\underline{\text { StanHeaders }}(\geq 2.18 .0)$, stats, graphics, methods

Imports: $\quad \underline{\text { coda, }}$ bnlearn, ggplot2, bayesplot, viridis, reshape2, $\underline{\text { dplyr }}$

Suggests: $\quad \underline{\text { loo }}(\geq 2.0 .0)$

Published: $\quad$ 2019-05-24

Author: $\quad$ Viet-Phuong La [aut, cre], Quan-Hoang Vuong [aut]

Maintainer: $\quad$ Viet-Phuong La $<$ lvphuong at gmail.com>

BugReports: $\quad$ https://github.com/sshpa/bayesvl/issues

License: $\quad \underline{\text { GPL }}(\geq 3)$.

URL: $\quad$ https://github.com/sshpa/bayesvl

NeedsCompilation: no

CRAN checks: $\quad$ bayesvl results

Downloads :

Reference manual: bayesvl.pdf

Package source: bayesvl 0.8.5.tar.gz

Windows binaries: r-devel: bayesvl 0.8.5.zip, r-release: bayesvl 0.8.5.zip, r-oldrel: bayesvl_0.8.5.zip

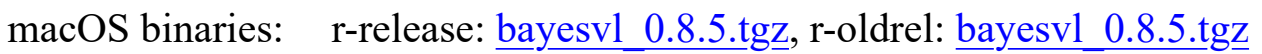

\section{Linking:}

Please use the canonical form https://CRAN.R-project . org/package=bayesvl to link to this page. 


\section{References}

La, V. P., \& Vuong, Q. H. (2019). bayesvl: Visually learning the graphical structure of Bayesian networks and performing MCMC with 'Stan'. The Comprehensive R Archive Network (CRAN). Available online: https://cran.r-project.org/package=bayesvl

Vuong, Q. H., \& La, V. P. (2019). BayesVL package for Bayesian statistical analyses in R. GitHub. Available online: https://github.com/sshpa/bayesvl

Vuong, Q. H., La, V. P., Nguyen, M. H., Ho, M. T., Ho, M. T., \& Mantello, P. (2020). Improving Bayesian statistics understanding in the age of Big Data with the bayesvl R package. Software Impacts, 4, 100016.

Vuong, Q. H., La, V. P., Nguyen, M. H., Ho, M. T., Tran, T., \& Ho, M. T. (2020). Bayesian analysis for social data: A step-by-step protocol and interpretation. MethodsX, 7, 100924. 